\title{
Das Sprachspiel Psychoanalyse und seine Regeln
}

\section{Diana Pflichthofer}

Online publiziert: 17. April 2013

(C) Springer-Verlag Berlin Heidelberg 2013

„Nur im Fluss des Lebens haben die Worte Bedeutung“

(Wittgenstein, Letzte Schriften zur Philosophie der Psychologie).

\section{The language game psychoanalysis and its rules}

In seinen Schriften zur Behandlungstechnik beschreibt Freud etliche für den psychoanalytischen Prozess konstitutive Regeln, die er als „Spielregeln“ verstanden wissen wollte, ,die ihre Bedeutung aus dem Zusammenhange des Spielplanes schöpfen müssen" (Freud 1913c, S.454). Hierin ist enthalten, dass sich die Bedeutung einer jeweiligen Regel nur aus dem dazugehörigen Kontext ergeben kann. Regeln sind für ein Spiel konstitutiv, das heißt, von ihrer Einhaltung wird es abhängen, ob das beabsichtigte Spiel zustande kommt. Über kurz oder lang kann man dann an neuralgische, schwierige Punkte geraten, die sich in jeder Spielpartie anders darstellen können.

Was für ein „Spiel“" aber wird in der Psychoanalyse ,gespielt“? Man könnte es mit Wittgenstein als ein besonderes Sprachspiel bezeichnen. Dieser Begriff ist bekanntlich in der späten Philosophie Wittgensteins von zentraler Bedeutung. Der Ausgangspunkt der Sprachspielanalogie ist dabei, dass es sich bei der Sprache um eine regelgeleitete Tätigkeit handelt. Anders aber als etwa strategische Regeln bestimmen die Regeln der Sprache nicht, ob die Züge erfolgreich sein werden, sondern ob sie sinnvoll sind (Glock 2000, S. 325 f.). Sinnvoll ist, was verstanden werden und Bedeutung erhalten kann. Mit der Sprachspielanalogie lenkte Wittgenstein die Aufmerksamkeit auf die menschliche Praxis, die alle unsere sprachlichen und nichtsprachlichen Tätigkeiten umfasst. Deren Ineinandergreifen sorgt für die hohe Komplexität der Sprach-

Dr. med. D. Pflichthofer $(\square)$

Uhlenhorster Weg 35, 22085 Hamburg, Deutschland

E-Mail: pflichthofer@t-online.de 
spiele und hebt diese von anderen Spielen ab. Sprachspiele sind immer in unsere jeweilige Lebenspraxis eingebettet. Wittgenstein spricht dabei von der Lebensform und meint damit „die Gesamtheit der Tätigkeit einer Sprachgemeinschaft“ (Glock 2000, S.330).

Dass es sich bei Psychoanalytikern um eine ganz eigene Sprachgemeinschaft handelt, spüren wir vermutlich alle, zum Beispiel immer dann, wenn uns ganz selbstverständliche und vertraute Begriffe von anderen nicht so verstanden werden, wie wir sie meinen. Nun sind wir als Psychoanalyse-Sprachgemeinschaft aber in eine größere allgemeine Sprachgemeinschaft eingebettet, bewegen uns auch in dieser. Problematisch, das heißt verwirrend wird es, so Wittgenstein, wenn Sprachspiele vermischt werden, wenn Wörter aus dem einen nach den Regeln eines anderen verwendet werden. Daraus ergibt sich die Konsequenz, dass den Teilnehmern an einem Sprachspiel bekannt sein sollte, welches Sprachspiel sie gerade spielen, damit sie sich verständigen können. Das jeweilige Sprachspiel, auch das psychoanalytische, beruht auf den je spezifischen Regeln.

Da es sich hier um Regeln der psychoanalytischen Praxis handelt, ist zur ErschlieBung von deren umfassender Bedeutung auch die Betrachtung der Praxis bzw. der durchaus unterschiedlichen Praxen notwendig. Das sagt oder schreibt sich leicht hin, ist aber doch in der Umsetzung nicht gar so einfach. Damit wir über unsere reale Behandlungs- und Sprachpraxis sprechen können, müssen bestimmte Voraussetzungen, bestimmte Rahmenbedingungen erfüllt sein.

Wann immer eine Psychoanalytikerin ${ }^{1}$ mit Kollegen über ihre reale (man könnte auch sagen wahrhafte) Behandlungspraxis spricht, tritt sie in einen Akt der Selbstreflexion ein. Damit ein solcher Prozess gelingt, ist es notwendig, dass auch die Zuhörenden (und die Mitlesenden), Mitdiskutierenden ihrerseits in einen solchen selbstreflexiven Prozess eintreten. In einer solchen Diskussion muss ein jeder frei sein, seine Sicht der Dinge darzustellen, ohne öffentlich oder auf andere Weise subtil dafür sanktioniert zu werden. (Auch die nach und nach hoffentlich in die Ecke geratende Zuschreibung ,unanalytisch“ im Rahmen einer solchen Diskussion ist eine Sanktion.) Der einen Fall vorstellende Kollege setzt sich, so er in einen selbstreflexiven Prozess eintritt, in besonderer Weise aus, weil ein solcher Prozess nicht möglich ist, ohne etwas von der eigenen Identität preiszugeben. Gerade das aber beansprucht einen Raum des besonderen Schutzes und Respekts.

Wenn die Wissenschaft lebendig gehalten werden und wenn die Psychoanalyse inmitten der zahlreichen anderen Therapieverfahren bestehen soll, muss die Theorie mit der Praxis abgeglichen werden. Eine Praxis ohne Theorie ist ebenso prekär wie eine Theorie, die sich nicht mit der Praxis vereinbaren lässt. Theorie und Praxis können wohl nie vollständig kongruent sein. Wenn sie jedoch zu weit voneinander abweichen, gerät eine Wissenschaft in allenfalls nur noch schwer lösbare Probleme. Daher ist die beständige Reflexion von Theorie und Praxis notwendig.

Passt sie in allen Belangen noch zu den heutigen Erkenntnissen? Ist es sinnvoll, an dieser oder jener Stelle etwas zu verändern, oder wäre das nur eine hypomanische Abwehr, die sich vom so genannten Zeitgeist bedrängt sieht?

\footnotetext{
${ }^{1}$ Es gelten selbstverständlich männliche und weibliche Form, auch wenn nur eine Form genannt sein sollte.
} 
Es ist daher sehr erfreulich, dass in diesem Heft einige Autoren einen Blick in ihre Praxis gewähren und sich den Lesern anvertrauen. Alle beschäftigen sich gleichermaßen mit „heiklen“ Themen innerhalb des Sprachspiels Psychoanalyse: der vakanten Sitzung und der Frage des Ausfallhonorars (Althoff), der Gegenübertragung in der Supervision (Herrmann), der negativen Übertragung und dem Respekt gegenüber dem Analytiker (Reisinger), dem Humor in der Psychotherapie (Frei) sowie der postanalytischen Beziehung (Pflichthofer).

Diese Themen lassen sich als „heikel“ bezeichnen, weil sie dazu angetan sind, mitunter zu affektiv recht aufgeladenen Diskussionen zu führen. Die Autoren begeben sich in ein Feld voller Unklarheiten, aber oft intensiver Affekte (jeder, der schon einmal das Thema „Ausfallhonorar“ mit einem Patienten bearbeitet hat oder lange einer sehr negativen Übertragung ausgesetzt war, wird das vermutlich nachvollziehen können) und schreiben über ihre Gedanken und Reflexionen zu den verschiedenen Themen.

Althoff fasst mit ihrem Aufsatz „Die vakante Sitzung und Supervision“ ein „heißes Eisen“" an und wirft die äußerst interessante Frage auf, ob womöglich die Verlässlichkeit der Analytikerin Einfluss auf die Häufigkeit der abgesagten Sitzungen seitens des Patienten hat, oder anders formuliert: Wenn sich die Analytikein verlässlich zeigt, ihrerseits wenige Stunden absagt, sagen dann auch die Patienten weniger Stunden ab? Zudem lässt Althoff den Leser an ihrer Vakanzregelung Anteil haben, derzufolge sie keine Absagefristen bespricht, den Patienten ihren nächsten anstehenden Urlaub mitteilt und im Übrigen schaut, was sich entwickelt. Und es entwickelt sich ganz offensichtlich eine Menge und eine Menge Unterschiedliches, wie die sehr anregenden Falldarstellungen zeigen.

Reisinger wendet sich ebenfalls zwei spannenden praktischen Aspekten psychoanalytischer Behandlungen zu. In seiner Arbeit „Negative Übertragung und Respekt gegenüber dem Analytiker" stellt er infrage, inwieweit eine heftige negative Übertragung zwangsweise eine Art „Gütesiegel“ für eine gelungene Behandlung sei, wie es den Autor manchmal anmutet, oder ob nicht gar die Gefahr besteht, der Analytiker werde zu einer Art „Watschenmann“ degradiert. Reisinger lässt den Leser an seiner Differenzierung zwischen „Sündenbock“ und „Watschenmann“ teilhaben: Während Ersterer von der Gruppe gefürchtet und daher in gewisser Weise respektiert werde, aber aufgrund seiner Gefährlichkeit aus der Gruppe ausgeschlossen werden solle, friste der „Watschenmann“ ein lächerliches und würdeloses Dasein, dessen Worte ohne Bedeutung blieben. Was nun, so fragt Reisinger, wenn der Analytiker in einer Behandlung in eine solche Rolle gerät? Auch Reisinger gewährt einen Einblick in seine psychoanalytische Praxis und in eine wahrlich schwer zu ertragende Behandlungskonstellation innerhalb einer 15 Jahre währenden Therapie und hält ein Plädoyer dafür, dass sich der Analytiker in bestimmten Situationen, in denen der Respekt vor ihm verloren gegangen zu sein scheint, diesen deutlich und bestimmt zurückerobern muss, soll die Behandlung nicht in einer Sackgasse stecken bleiben.

Frei wendet sich in seinem interessanten Beitrag einem Thema zu, von dem man nicht behaupten kann, dass es in der Psychoanalyse inflationär behandelt würde. Seine Arbeit „Humor, Kreditierung und Vertrauensaufbau in einem Erstgespräch nach Suizidversuch“ beginnt er mit einem wichtigen, tatsächlich eher vernachlässigten Gedanken: Während durchaus häufiger gesagt wird, dass der Patient doch 
dem Analytiker, dem Therapeuten vertrauen müsse, dieser Vertrauensvorschuss geradezu elementar für eine erfolgreiche Behandlung sei, wendet Frei sein Augenmerk auf jenen Vertrauensvorschuss, den der Analytiker seinem Patienten gewähren muss, indem er nämlich Vertrauen in das Entwicklungspotenzial des Patienten setzt. Anhand einer sehr spannenden Fallvignette, in der die Therapeutin eine paradoxe Intervention vornimmt, erläutert Frei dieses Prinzip der „Kreditierung“ und stellt den Nutzen und die Notwendigkeit von Humor in menschlichen Beziehungen heraus. Humor sei eine lebensbejahende Haltung, bei der die Realitätsprüfung intakt bleibe, der nicht sadistisch sei, sondern dem Individuum zur Selbstbehauptung gegenüber einer frustrierenden Realität verhelfe.

Herrmann befasst sich in seinem Beitrag ,Supervision zwischen Differenz und Triangulierung. Mit der Gegenübertragung arbeiten“ mit Aspekten, die dabei helfen können, Supervisionsprozesse zu verstehen, in denen es um die differierenden Sichtweisen der Beteiligten geht und die zugleich innerhalb eines triadischen Settings stattfinden. Dabei geht er von seiner Erfahrung aus, dass die Triangulierung in der Supervision eine Differenzierung ermöglicht, in der vor allem an der Gegenübertragung des Behandlers gearbeitet werden kann. Hermann bezieht sich dabei auf Klüwer, der beklagt habe, dass Supervision etwas sei, dass eher gemacht als diskutiert würde. Auch Herrmann gewährt Einblicke, dieses Mal in eine Supervisionstunde. Anhand der Schilderung einer Supervisionssequenz legt er die aus seiner Sicht wichtigen und wirksamen Aspekte supervisorischer Arbeit und gemeinsamen Reflektierens innerhalb der asymmetrischen Beziehungsform „Supervision“ dar.

Das vorliegende Heft enthält außerdem zwei freie Originalarbeiten, die eng mit dem Thema „Spielregeln“ assoziiert sind: Carl Nedelmann beschäftigt sich in seinem Aufsatz „Die allgemeine Grundlage der psychotherapeutischen Behandlungstechnik auf psychoanalytischer Basis" mit den Gemeinsamkeiten von tiefenpsychologisch fundierter Psychotherapie, psychoanalytischer Psychotherapie und Psychoanalyse im engeren Sinne. Aus der Sicht des Autors stellen die beiden erstgenannten Verfahren unterschiedliche Anwendungsformen der Psychoanalyse dar.

Leopold Morbitzer diskutiert in seinem Aufsatz „Zur Psychoanalyse des Glücks“ das Glücksstreben und Glückserleben aus der Perspektive verschiedener psychoanalytischer Theorien. Das Märchen von „Hans im Glück“ begleitet ihn bei seinen Überlegungen.

Das Heft schließt mit einem Beitrag, in dem Gedanken über das Ende der Psychoanalyse und die postanalytische Beziehung mitgeteilt werden. Jede Analyse hört irgendwann auf und geht in eine Zeit „danach“ über. Zu lesen ist darüber (noch) wenig.

Die in dieser Ausgabe vom Forum der Psychoanalyse gewährten Einblicke in die Praxis sind für alle Psychoanalytiker von großem Wert; daher gebührt all jenen Kollegen Dank, die ihre Fragen, Gedanken, Unsicherheiten, Enttäuschungen und Erfolge mit den Lesern teilen. Sie stellen etwas von sich zur Verfügung und sie eröffnen den Raum für Diskussionen, den das Forum der Psychoanalyse auch gerne bereitstellen möchte. Daher soll an dieser Stelle noch einmal an die Rubriken „Diskussionsforum“ und „Kommentare“ erinnert werden, Plattformen, auf denen Kollegen ihre Gedanken zu dem von den Autoren Geäußerten darlegen, Anregungen, Bestätigungen, Differenzen mitteilen können. Selbstreflexion ist ein Prozess, der nie zu einem Abschluss 
kommt; er wächst gewissermaßen mit der Analytikein mit. Dennoch sind wir immer wieder gezwungen, Entscheidungen zu treffen, auch in jeder Stunde. Sagen wir etwas? Schweigen wir? Was sagen wir? Daran führt kein Weg vorbei und diese Entscheidungen fließen - wenn es gut geht - in den selbstreflexiven Prozess mit ein. Regeln können helfen, sich zu orientieren, zugleich sind sie eine ständige Herausforderung für den selbstreflexiven Prozess - oder sollten es wenigstens sein.

Der Wiener Philosoph Karl Popper wendet sich in seinem Buch Auf der Suche nach einer besseren Welt gegen die These, dass die Objektivität einer Wissenschaft von der Objektivität des einzelnen Wissenschaftlers abhänge. Diesem ist es inzwischen anerkanntermaßen niemals möglich, objektiv zu sein, auch dem Naturwissenschaftler nicht, auch nicht jenem, der Daten erhebt und sie vermeintlich „objektiv“ auswertet. Sie alle, wir alle sind - wenn man so will - befangen, befangen durch die eigene Subjektivität. Dennoch kann eine Wissenschaft nach Objektivität streben, und deren Objektivität liege, so Popper, in der kritischen Tradition, und zwar ,in jener Tradition, die es trotz aller Widerstände so oft ermöglicht, ein herrschendes Dogma zu kritisieren“ (Popper 2011, S. 88). Daraus ergibt sich folgender Schluss:

... die Objektivität der Wissenschaft ist nicht eine individuelle Angelegenheit der verschiedenen Wissenschaftler, sondern eine soziale Angelegenheit ihrer gegenseitigen Kritik, der freundlich-feindlichen Arbeitsteilung der Wissenschaftler, ihres Zusammenarbeitens und auch ihres Gegeneinanderarbeitens. (Popper 2011, S. 88)

Die Psychoanalyse ist eine großartige Entdeckung, die sich im Laufe der Zeit verändert hat. Sie wird von Menschen ausgeübt und ist dazu da, Menschen zu helfen. Damit ihr das gelingen kann, muss sie als Wissenschaft lebendig bleiben, indem also die ihr Angehörenden Meinungen, Thesen und Kritik ${ }^{2}$ hören und sie ebenso äußern.

Der Austausch über die reale Praxis, über Regeln, Vertrautes, Unvertrautes und private Theorien der Psychoanalyse könnte etwas dazu beitragen.

\section{Literatur}

Freud S (1913c) Zur Einleitung der Behandlung. Gesammelte Werke, Bd8. Fischer, Frankfurt a. M., S $454-478$

Glock H-J (2000) Wittgenstein-Lexikon. Wissenschaftliche Buchgesellschaft, Darmstadt (1996)

Popper KR (2011) Auf der Suche nach der besseren Welt. Vorträge und Aufsätze aus dreißig Jahren. Piper, München (1987)

\footnotetext{
${ }^{2}$ Kritik, so sieht Popper sie, ,zügelt die Phantasie, ohne sie zu fesseln“ (Popper 2011, S. 67).
} 